\title{
Screening for Down's syndrome in the North East Thames Region
}

\author{
VICTORIA MURDAY, JOAN SLACK
}

\begin{abstract}
The suggested strategies for a screening programme for Down's syndrome by maternal serum $\alpha$ fetoprotein concentration were examined and tested on the experience of the North East Thames Regional. Screening by maternal serum $\alpha$ fetoprotein concentration may be used to identify pregnancies at increased risk, but this is useful only in women aged over 32 whose collective risk is greater than one in $\mathbf{2 0 0}$.

The absolute probability of carrying babies with Down's syndrome for individuals in this high risk group can then be calculated and used to decide whether further diagnosis by amniocentesis is desired.
\end{abstract}

\section{Introduction}

The North East Thames regional screening policy for Down's syndrome is to offer amniocentesis to all women who will be 38 at their expected date of delivery, as well as offering it to those couples who have had a child with a chromosome abnormality or are known to carry a balanced translocation.

The observation by Merkatz et al and Cuckle et al that pregnancies resulting in babies with Down's syndrome are associated with reduced maternal serum $\alpha$ fetoprotein concentration ${ }^{12}$ has prompted a review of the North East Thames regional screening programme for Down's syndrome. In particular it might be possible to identify younger mothers who are at increased risk who could be offered amniocentesis. To assess the likely effects of increasing the scope of the present programme, however, the numbers affected, the likely uptake, and the expected results must be examined.

We ascertained all the babies with Down's syndrome born in North East Thames Regional Health Authority from 1 April 1982 to 31 March 1983 and examined the effects of the screening programme from 1 January 1982 to 31 December 1982 . We have used this cohort to examine the possible effects of using screening by maternal serum $\alpha$ fetoprotein concentration in the strategies suggested by Cuckle $e t a l^{2}$ to identify younger mothers at increased risk of having a baby with Down's syndrome and to calculate absolute probabilities in the mothers at risk so that the decision whether to offer and accept amniocentesis could be based on their individual risks.

\section{Methods}

Three regional cytogenetic laboratories were asked to notify any babies with Down's syndrome born from 1 April 1982 to 31 March 1983. All paediatricians, obstetricians, and district medical officers in the region gave information about the pregnancies and births of the babies with Down's syndrome. None refused. Some replied personally, and most allowed us to see hospital records. Seventy eight babies with Down's syndrome were identified.

\footnotetext{
Department of Clinical Genetics, Royal Free Hospital, London NW3 2QG, and Royal College of Physicians Research Unit, London

VICTORIA MURDAY, BSC, MRCP, clinical research fellow, Imperial Cancer Research Fund and honorary senior registrar

JOAN SLACK, DM, FRCP, senior lecturer and honorary consultant in clinical genetics

Correspondence to: Dr J Slack, Department of Clinical Genetics, Royal Free Hospital, London NW3 2QG.
}

Details of the karyotypes of all the amniocenteses performed in 1982 and on liveborn babies with Down's syndrome born from April 1982 to March 1983 were supplied by the cytogenetic laboratories. Maternal serum $\alpha$ fetoprotein concentrations in the pregnancies that resulted in liveborn infants and that resulted in termination because of Down's syndrome were sought from the six laboratories in the region carrying out the measurements; 45 were available.

Gestational age at the time of sampling for maternal serum $\alpha$ fetoprotein concentration was based on dates of the last menstrual period. In two pregnancies with Down's syndrome there was a discrepancy for the gestational age between the dates of the last menstrual period and findings on ultrasound scan, so the gestational date according to the scan was used. The maternal serum $\alpha$ fetoprotein concentration was expressed in multiples of the median for the appropriate gestational age and for each laboratory at the time of measurement to allow for interlaboratory comparisons. For each liveborn baby with Down's syndrome 10 controls were obtained, excluding those with congenital abnormalities known to be associated with raised maternal serum $\alpha$ fetoprotein concentrations. The controls were taken from the same period and matched for the laboratory and for gestational age in 44 of the 45 cases. Finding sufficient controls from one laboratory for gestational age of 19 weeks proved impossible, so an additional 10 were obtained with gestational age of 18 weeks. The maternal serum $\alpha$ fetoprotein concentration on the control pregnancies and pregnancies with Down's syndrome were expressed as log percentages of the median as maternal serum $\alpha$ fetoprotein concentration is distributed log normally. The maternal serum $\alpha$ fetoprotein concentrations in control pregnancies and pregnancies with Down's syndrome were compared using unpaired Student's $t$ test.

\section{Results}

Sixty four babies were live born with Down's syndrome. Sixty three of these babies were notified by the laboratories, 37 by obstetricians and paediatricians, 15 by the community health services, and one, who was not identified as having Down's syndrome until four months after the end of the ascertainment, was referred to a genetic clinic. Of these 64 babies, 62 were regular trisomy 21 and two were de novo 21/21 Robertsonian translocations. In 198214 pregnancies with Down's syndrome were detected and terminated, all of which were regular trisomy 21 .

Overall, 1396 births were recorded and 691 amniocenteses performed in women of 38 and over. Of these, 55 births were in women over 45,11 of whom received amniocentesis.

In the 691 mothers who were screened for raised maternal age, 11 fetuses were detected with Down's syndrome, and 11 other chromosome abnormalities were found. All pregnancies except one, with triple X karyotype, were terminated. A further $\mathbf{4 8 0}$ amniocenteses were performed for other indications, such as raised maternal serum $\alpha$ fetoprotein concentration, family history of chromosome abnormality, and a variety of other obstetric indications, such as polyhydramnios, and resulted in the termination of a further three pregnancies with Down's syndrome and six fetuses with other chromosome abnormalities.

Forty four (69\%) maternal serum $\alpha$ fetoprotein concentrations were identified in the 64 liveborn babies with Down's syndrome, measured between 15 and 19 weeks, and one in a mother who had a fetus with trisomy 21 terminated. Figure 1 shows the values of maternal serum $\alpha$ fetoprotein

TABLE I-Maternal serum $\alpha$ fetoprotein concentrations and log $\%$ multiples of the median in pregnancies with Down's syndrome and control pregnancies

\begin{tabular}{lcc}
\hline & Control pregnancy & $\begin{array}{c}\text { Pregnancy with } \\
\text { Down's syndrome }\end{array}$ \\
\hline $\begin{array}{l}\text { No } \\
\begin{array}{l}\text { Median in multiples } \\
\text { of the median }\end{array}\end{array}$ & 450 & 45 \\
$\begin{array}{l}\text { Mean log \% in multiples } \\
\text { of the median }\end{array}$ & 0.99 & 0.63 \\
Standard deviation & 1.98 & $1.82^{\star}$ \\
& 0.1982 & 0.202
\end{tabular}

${ }^{\star} \mathrm{p}<0 \cdot 001$. 
concentrations in multiples of the median plotted on a log scale. The median maternal serum $\alpha$ fetoprotein concentrations in the pregnancies with Down's syndrome and in the control pregnancies were 0.63 and 0.99 , respectively.

Table I shows the median maternal serum $\alpha$ fetoprotein concentrations in pregnancies with Down's syndrome and control pregnancies and the mean and standard deviation in log percentage multiples of the median. The difference was significant $(p<0 \cdot 001)$. Table II shows the distribution of maternal serum $\alpha$ fetoprotein concentrations in multiples of the median in pregnancies with Down's syndrome and control pregnancies.

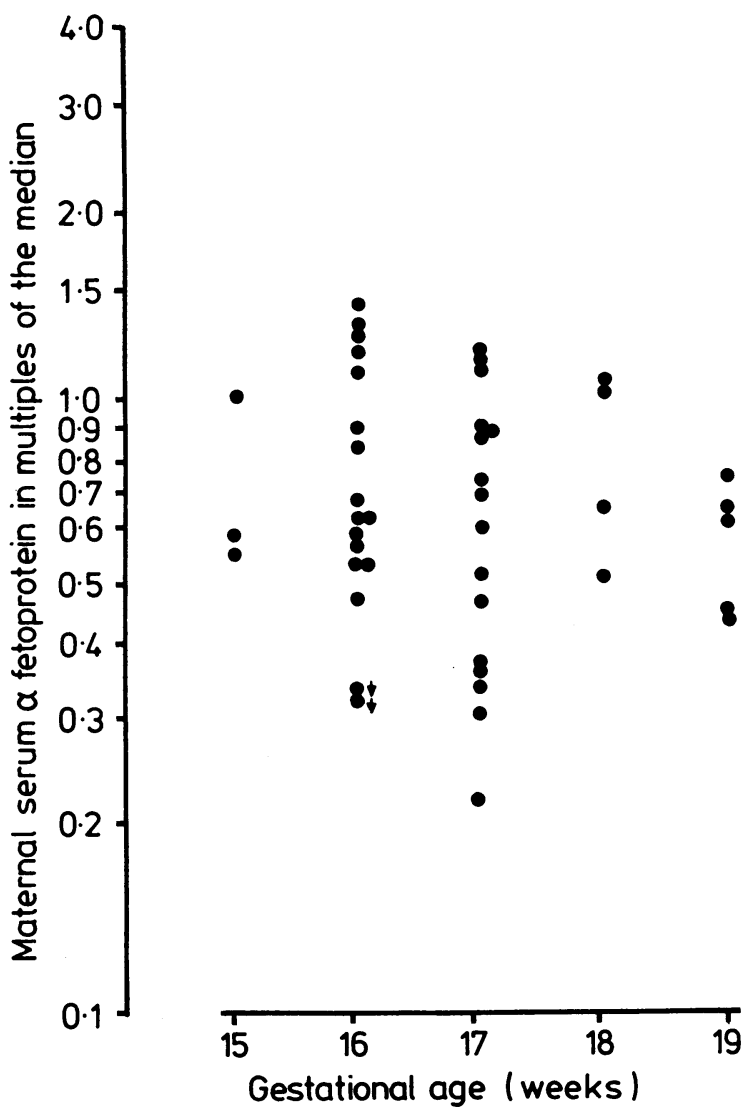

FIG 1-Maternal serum a fetoprotein in multiples of median on $\log$ scale in mothers of babies with Down's syndrome by gestational date.

TABLE II-Distributions of maternal serum $\alpha$ fetoprotein concentrations in multiples of the median in pregnancies with Down's syndrome and control pregnancies

\begin{tabular}{lccc}
\hline $\begin{array}{c}\alpha \text { Fetoprotein } \\
\text { concentration } \\
\text { (Multiple of the median) }\end{array}$ & $\begin{array}{c}\text { Pregnancy with } \\
\text { Down's syndrome } \\
(\%)\end{array}$ & $\begin{array}{c}\text { Control } \\
\text { pregnancy } \\
(\%)\end{array}$ & $\begin{array}{c}\text { Relative } \\
\text { risk }\end{array}$ \\
\hline$\leq 0.4$ & $15 \cdot 55$ & 3.55 & 4.38 \\
$\leqslant 0.5$ & 24.44 & 6.89 & 3.547 \\
$\leqslant 0.6$ & 42.22 & $14 \cdot 22$ & 2.969 \\
$\leqslant 0.7$ & 60 & $21 \cdot 77$ & 2.76 \\
$\leqslant 0.8$ & 64 & $32 \cdot 22$ & 1.986 \\
$\leqslant 0.9$ & $75 \cdot 55$ & 44 & 1.717 \\
$\leqslant 1.0$ & 80 & 52 & 1.5385 \\
\hline & 100 & 100 & 1
\end{tabular}

\section{Discussion}

In 1982 and 1983 the programme of amniocentesis for all mothers of 38 and over at the expected date of delivery in North East Thames Regional Health Authority reached only $49 \%$ of the mothers at risk and only $20 \%$ of the women of 45 and over. Only 11 of the 19 babies with Down's syndrome born to mothers of 38 and over at the expected date of delivery were identified. In the study year $18 \%$ of all babies with Down's syndrome born in North East Thames Regional Health Authority were detected. In contrast maternal serum $\alpha$ fetoprotein screening was carried out in 44 of a possible 58 mothers in the study (six mothers attended hospitals where maternal serum $\alpha$ fetoprotein screening was not performed). During 1982, $89 \%$ of women eligible for screening by maternal serum $\alpha$ fetoprotein received the test in the North East Thames Regional Health Authority (personal communication from Dr Marcus Pembrey). The poor uptake of the amniocentesis programme might in part be because of anxiety of the risk of miscarriage, which is now thought to be less than $0.5 \%$, or because screening might be less acceptable to older mothers. Some couples refused screening on ethical grounds, some were not informed about the opportunity for screening, and some did not fully understand the explanations offered because of language difficulties. Six out of eight babies with Down's syndrome born to mothers over 38 belonged to ethnic minority groups and five of these were not offered screening, one having booked too late.

As $56(88 \%)$ of the babies with Down's syndrome were born to younger mothers outside the present screening programme, other women need to be identified who may be at increased risk. We confirmed the findings of Cuckle $e t$ al and of six out of seven studies quoted by Spencer and Carpenter, which found that maternal serum $\alpha$ fetoprotein concentration was reduced in mothers of babies with Down's syndrome, ${ }^{23}$ and examined the findings retrospectively to see whether we could have used them to identify babies with Down's syndrome born in the North East Thames Regional Health Authority, using the three alternative strategies suggested by Cuckle $e t a l^{2}$ and detailed below.

Firstly, if amniocentesis was introduced for all mothers of 35 and over, a further eight fetuses with Down's syndrome could have been identified by 2666 amniocenteses, or one such fetus in every 333 amniocenteses. Secondly, if $89 \%$ of mothers aged under 38 had maternal serum $\alpha$ fetoprotein screening then 3014 would have levels equal to or less than 0.5 multiples of the median and a further nine fetuses with Down's syndrome could have been identified, or 1 Down's in every 334 amniocenteses. Thirdly, if $89 \%$ of the mothers aged 25-37 received maternal serum $\alpha$ fetoprotein screening and had been offered amniocentesis according to the suggested sliding scale of reduced cut off concentrations of maternal serum $\alpha$ fetoprotein with decreasing maternal age (table III) a further 12 babies with Down's syndrome could have been detected by 3090 amniocenteses, or one in every 258 amniocenteses. The effectiveness of this strategy diminishes in the younger age groups so that only two out of 15 babies with Down's syndrome born to mothers aged between 25 and 31 who had had maternal serum $\alpha$ fetoprotein screening would have been identified compared with 10 out of 17 born to mothers aged between 33 and 37 .

The risk of Down's syndrome in a pregnancy with maternal serum $\alpha$ fetoprotein concentration at or below a given concentration in a woman of known maternal age may be calculated by combining the maternal age risk with the relative risk associated with maternal serum $\alpha$ fetoprotein. The relative risk is the ratio of areas under the frequency distribution curves and below the chosen cut off point in mothers of babies with Down's syndrome and controls (table II).

TABLE III-Pregnancies with Down's syndrome in North East Thames Regional Health Authority in 1982-3 identified by low maternal serum a fetoprotein using sliding Health
scale

\begin{tabular}{|c|c|c|c|c|c|}
\hline $\begin{array}{l}\text { Maternal } \\
\text { age }\end{array}$ & $\begin{array}{l}\text { No of } \\
\text { births }\end{array}$ & $\begin{array}{l}\text { No of babies } \\
\text { born with } \\
\text { Down's } \\
\text { syndrome }\end{array}$ & $\begin{array}{l}\text { No of mothers } \\
\text { of babies with } \\
\text { Down's syndrome } \\
\text { with maternal } \\
\text { serum } \alpha \text { feto- } \\
\text { protein concen- } \\
\text { tration measured } \\
\text { (gestational age } \\
15-19 \text { weeks) }\end{array}$ & $\begin{array}{l}\text { Sliding scale cut } \\
\text { off level of } \alpha \\
\text { fetoprotein } \\
\text { concentration in } \\
\text { multiples of the } \\
\text { median }\end{array}$ & $\begin{array}{l}\text { Babies with } \\
\text { Down's } \\
\text { syndrome } \\
\text { identified }\end{array}$ \\
\hline $\begin{array}{l}37 \\
36 \\
35 \\
34 \\
32-33 \\
25-31\end{array}$ & $\begin{array}{rr} & 636 \\
& 846 \\
1 & 184 \\
1 & 499 \\
3 & 720 \\
22 & 237\end{array}$ & $\begin{array}{r}2 \\
3 \\
3 \\
7 \\
10 \\
20\end{array}$ & $\begin{array}{r}1 \\
2 \\
3 \\
3 \\
8 \\
15\end{array}$ & $\begin{array}{l}\leqslant 1.0 \\
\leqslant 0.9 \\
\leqslant 0.8 \\
\leqslant 0.7 \\
\leqslant 0.6 \\
\leqslant 0.5\end{array}$ & $\begin{array}{l}1 \\
1 \\
3 \\
3 \\
2 \\
2\end{array}$ \\
\hline Total & 30122 & 45 & 32 & & 12 \\
\hline
\end{tabular}

\section{$\underset{\sim}{0}$}

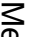
2 . .

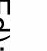

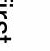
. . $\overrightarrow{0}$

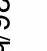

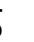
. 윽

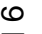


The risk for each maternal age over 25 has been calculated by combining data from Sutherland et al from Australia ${ }^{4}$ and Hook and Lindsiö from Sweden' on the birth incidence of babies with Down's syndrome and the combined risks of women of 25 years and over the maternal serum $\alpha$ fetoprotein equal to or less than multiples of the median (fig 2). The risk for women of 38 at the expected date of delivery in the Swedish and Australian series was one in 220, and this risk combined with the relative risk for women with lower maternal serum $\alpha$ fetoprotein concentrations is shown for comparison.

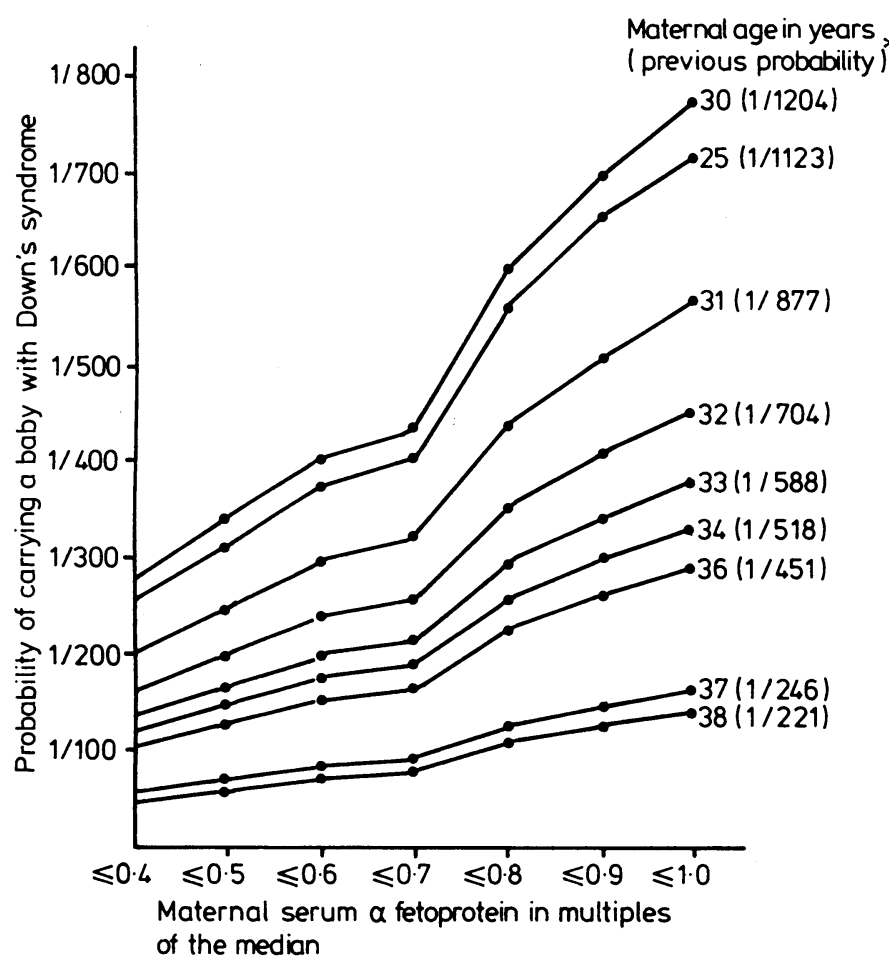

FIG 2-Probability of carrying babies with Down's syndrome at or below maternal serum $\alpha$ fetoprotein concentrations by maternal age at expected date of delivery. ( ${ }^{\star}$ Probability calculated by two previous reports. ${ }^{45}$ )

For women of 31 and less the risk is not greater than one in 200 even in those with maternal serum $\alpha$ fetoprotein $\leqslant 0.05$ multiples of the median. At 31 and less, therefore, the sliding scale suggested by Cuckle $e t a l^{2}$ fails to provide a cut off point below which the level of risk is acceptable for the North East Thames Regional Health Authority screening programme. It is possible, however, to select a cut off point of maternal serum $\alpha$ fetoprotein concentration for women who will be 32 or more at the expected date of delivery to identify a group of women whose overall risk of having a baby with Down's syndrome is one in 200 (see fig 2).

Any retrospective study such as that of Spencer and Carpenter ${ }^{3}$ that has included women less than 32 years of age, when the greatest number of pregnancies occur, will therefore show that many amniocenteses will have been performed and a low rate of detection achieved. Our findings were different because not only did they include women in the younger age groups but also the overall incidence of Down's syndrome in their population seemed to be exceptionally low. In their series there were 36 babies with Down's syndrome ascertained from 29540 pregnancies, an incidence of one in 821 compared with the incidence in our study of one in 674 in 1982-3 and one in 678 reported from the combined studies from Australia and Sweden. ${ }^{45}$ This discrepancy could be explained by incomplete ascertainment by the laboratory at Oldchurch Hospital but might also be caused by special features of the maternal age distribution in the Barking, Havering, and Brentwood district population from which their patients are drawn.

Comparison of the maternal age distribution in 1982-3 in Barking, Havering, and Brentwood district with the North East
Thames Regional Health Authority showed that $37 \%$ of mothers were between 25 and 29 in the district compared with $34 \%$ in the region, and that only $0.09 \%$ of mothers were between 40 and 45 in the district compared with $1.21 \%$ in the region. Overall, the distortion of the maternal age distribution in the Barking, Havering, and Brentwood district would account for a deficit of at least six babies with Down's syndrome in the year 1982-3, and if this discrepancy had been present throughout the four years of the period of the Oldchurch study the effect on their figures would have been compounded. This might amply account for their claim to have shown "high fetal risk, poor fetal yield" and their conclusion that screening based on low maternal serum $\alpha$ fetoprotein "is almost certainly unacceptable."

In our series, if the sliding scale is applied only to women who had maternal serum $\alpha$ fetoprotein measured and who are 32 and over at the expected date of delivery (given an $89 \%$ uptake) and where the risk exceeds one in 200, a total of 1726 amniocenteses would have identified 10 babies with Down's syndrome or 1 in 173 amnios. If only half the mothers took up the offer of amniocentesis, as in the women over 38 , roughly 860 extra amniocenteses would have been performed in the North East Thames Regional Health Authority in 1982-3 for low maternal serum $\alpha$ fetoprotein.

This strategy would be the most efficient as a screening programme and could be implemented with reasonable increases in antenatal and cytogenetic services. The number of amniocenteses could be further reduced, as suggested by Cuckle $e t a l$, by a repeat measurement of maternal serum $\alpha$ fetoprotein concentration and accurate dating by ultrasound ${ }^{6}$ of those picked up by the first screening procedure. ${ }^{2}$ Furthermore, as Down's syndrome is very rare in babies born to mothers with a maternal serum $\alpha$ fetoprotein concentration greater than 2.5 multiples of the median (Cuckle estimated one in 6000 in a personal communication), and none of the pregnancies with Down's syndrome in our study had maternal serum $\alpha$ fetoprotein greater than 1.5 multiple of the median, it should be possible to reduce the karyotyping on amniotic fluid from younger mothers investigated for raised maternal serum $\alpha$ fetoprotein concentration.

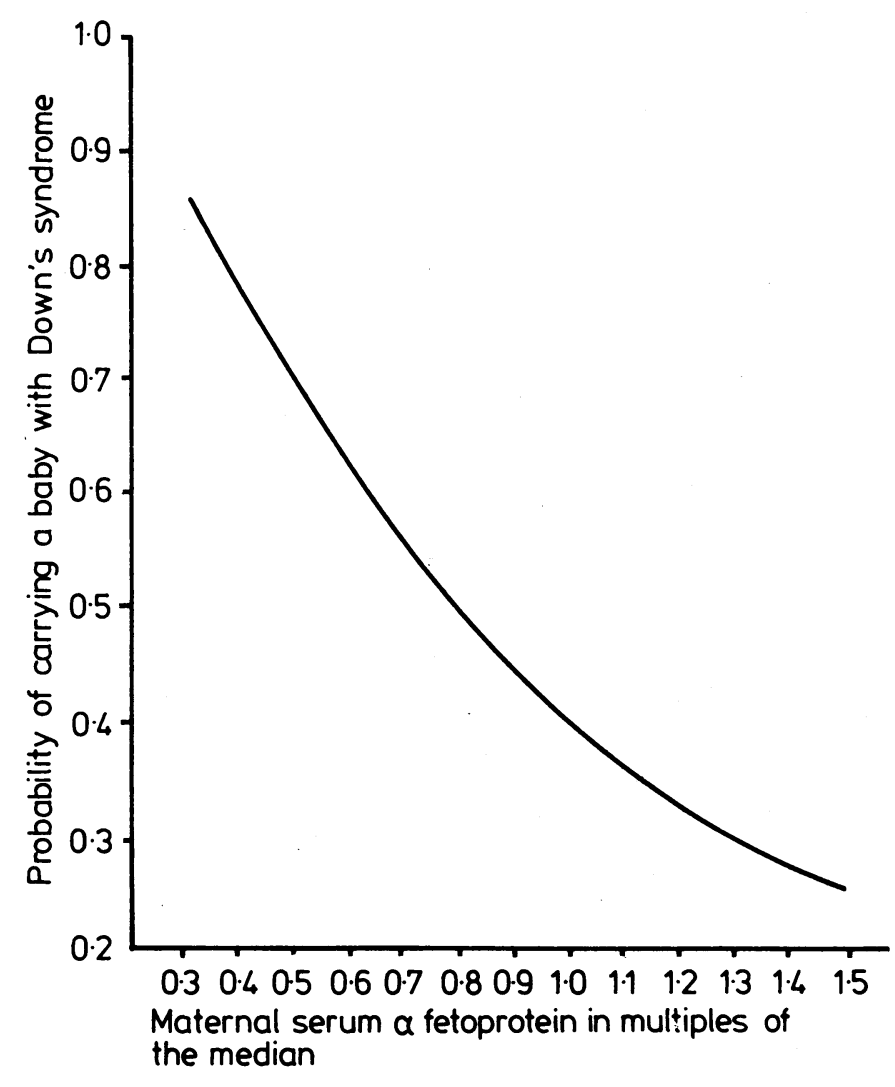

FIG 3-Probability of carrying babies with Down's syndrome at maternal serum $\alpha$ fetoprotein concentrations between 0.3 and 1.5 multiples of median. 


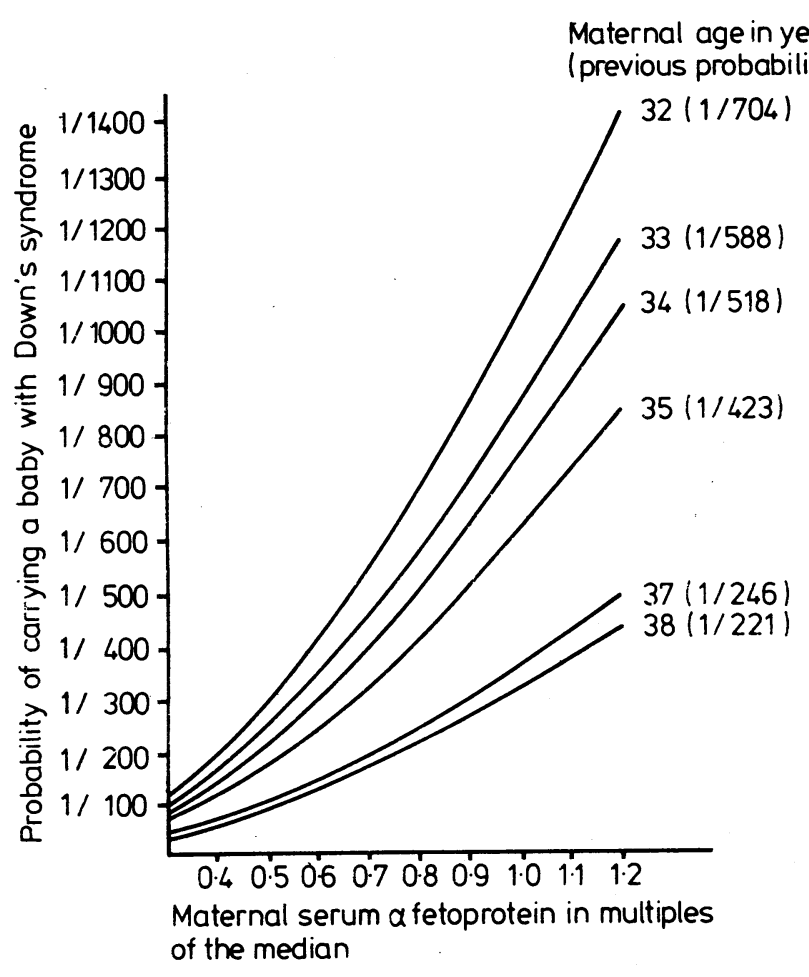

FIG 4 Absolute probability of carrying babies with Down's syndrome at maternal serum $\alpha$ fetoprotein concentrations according to maternal age at expected date of delivery. ( $\star$ Probability calculated by two previous reports. ${ }^{+5}$ )

With a screening programme to identify a group of mothers at increased risk of a pregnancy with Down's syndrome, it is possible to calculate individual risks or absolute probabilities on which decisions for or against further diagnostic procedures can be based. The absolute probability of an individual woman with known maternal serum $\alpha$ fetoprotein concentration and age at expected date of delivery can be calculated by the method described by Dennis and Carter for overlapping normal distributions. ${ }^{7}$ Figure 3 shows the probability of a mother carrying a baby with Down's syndrome with maternal serum $\alpha$ fetoprotein concentration between 0.3 and 1.5 multiples of the median where the probability of carrying a baby with Down's syndrome is one using the distributions shown in table II. Figure 4 shows the absolute probability of carrying a baby with Down's syndrome calculated for individual values of maternal serum $\alpha$ fetoprotein by maternal age.

We therefore suggest that maternal serum $\alpha$ fetoprotein concentration equal to or less than the sliding scale described by Cuckle $t$ $a l^{2}$ should be used as a screening test to identify women whose collective risk of having a baby with Down's syndrome is greater than one in 200 , and these will be the women aged 32 and over. The accuracy of the information on the high risk group could then be improved by repeat maternal serum $\alpha$ fetoprotein screening and scan. Their absolute probability of carrying a baby with Down's syndrome could then be calculated using the aforementioned probability at the individual maternal age and an informed decision about further diagnostic tests could be based on a known risk of carrying a baby with Down's syndrome, the views of the parents, and the risks of the procedure.

We thank the obstetricians, paediatricians, and district medical officers and the North East Thames Regional Cytogenetic and AFP laboratories for their cooperation and Professor Timothy Chand for his help in collecting MSAFP data. We thank the Royal College of Physicians Research Unit for their help and encouragement and Mrs Joy Robertson for secretarial help.

\section{References}

Merkatz IR, Nitowsky HM, Macri JN, Johnson WE. An association between low maternal serum alpha-fetoprotein and fetal chromosomai abnormalities. Am $\mathcal{J}$ Obstet Gynecol 1984;14:886-92. Cuckle HS, Wald NJ, Lindenbaum RH. Maternal serum alpha-fetoprotein measurements: a screening test for Down's syndrome. Lancet 1984;i:926-9.

Spencer K. Carpenter P. Screening for Down's syndrome using serum $\alpha$ fetoprotein: a retrospective study indicating caution. Br Med f 1985;290:1940-3.

Sutherland GR, Clisby SR, Blood G, Carter RF. Down's syndrome in South Australia. Med f Aust 1979;2:58-61

5 Hook EB, Lindsjö A. Down's syndrome in livebirths by single year maternal age interval in a Swedish study: comparison with results from a New York State study. Am $\mathcal{J}$ Hum Genet 1978;30:19-27.

6 Cuckle HS, Wald NI, Lindenbaum RH. Screening for Down's syndrome using serum a fetoprotein. BrMed f 1985;291:349.

Dennis NR, Carter CO. Use of overlapping normal distributions in genetic counselling. 7 Med Genet 1978;15:106-8.

Accepted 25 September 1985)

\section{SHORT REPORTS}

\section{Safety of handling cytotoxic agents: a cause for concern by pharmaceutical companies?}

The increased usage of cytotoxic agents has been accompanied by growing concern for the safety of those who manufacture, prepare, and administer these drugs or dispose of waste products from them. The immediate risk is vesicant damage to skin or membranes from contact with these agents. Less tangible is the possibility of carcinogenic damage to those handling these drugs. Although patients receiving therapeutic doses are probably at risk of carcinogenesis, ${ }^{1}$ a similar case has not been established for those concerned in their preparation and administration. ${ }^{2}$ Much interest has been aroused by the observation that in vitro mutagenesis in bacteria may be induced by the urine of nurses working with these drugs. The relevance of this finding, if any, and even its reproducibility, however, are disputed. ${ }^{2}$ Nevertheless, as carcinogenesis is a long term putative hazard it is clear that cytotoxic agents should be handled with extreme care. ${ }^{3}$ I have reviewed the information and advice available.

\section{Present study}

Wearing gowns, gloves, and goggles, whether or not a vertical laminar flow cabinet is used, seem appropriate and useful measures when handling cytotoxic agents. Prudent management of spillage and excreta is indicated, and incineration of such materials at as high a temperature as possible also seems useful. Many different institutions and authorities have prepared guidelines and made practical suggestions concerning the preparation, administration, and disposal of cytotoxic agents. ${ }^{24}$

Presumably the pharmaceutical companies have informatioon and interest in such safeguards and might pass on information to users. Certainly their drug package inserts are informative on chemical constitution, physical properties, indications for usage, dosage, contraindications, and advice on dealing with adverse reactions in patients. Twenty four such inserts relating to the 21 most commonly used agents (table) were inspected. Nine intimated that the drugs were irritant to skin and mucous membranes, but only four recommended wearing gloves and only three mentioned eye hazards. Preparation of drugs in a vertical laminar flow safety cabinet, which is recommended by many ${ }^{4}$ but not all authorities, was not mentioned in any case.

The question of disposal of residue, waste, and spillage is a vexed one and advice of the manufacturers has been sought in the past. Evidently the general opinion was that flushing the drug residue through the drainage system with copious amounts of water was adequate, ${ }^{3}$ but this was not reiterated in any drug insert inspected. Other experts have suggested that solid waste materials should be buried or incinerated at around $1000^{\circ} \mathrm{C},{ }^{5}$ but only two inserts touched on this subject.

There is a possibility that patients receiving cytotoxic agents may excrete appreciable amounts of these drugs and that their excreta may be hazardous. Whether this is true in the usual case is doubtful but it may be so in those given very high doses and those receiving treatment by bladder instillation. This particular problem is not covered in the world publications at large or in any company packaging literature. 